\title{
Ubiquitin-Specific Peptidase USP22 Negatively Regulates the STAT Signaling Pathway by Deubiquitinating SIRT1
}

\author{
Ning Ao Yanyan Liu Hailiang Feng Xiaocui Bian Zhanwen Li Bei Gu \\ Xiaomei Zhao Yuqin Liu \\ Department of Pathology, Institute of Basic Medical Sciences, Chinese Academy of Medical Sciences \\ and Peking Union Medical College, Tsinghua University, Beijing, China
}

\author{
Key Words \\ USP22 • SIRT1 • STAT3 • Deubiquitinating • Acetylation
}

\begin{abstract}
Background/Aims: The ubiquitin-specific peptidase USP22 mediates various cellular and organismal processes, such as cell growth, apoptosis, and tumor malignancy. However, the molecular mechanisms that regulate USP22 activity remain poorly understood. Here we identify STAT3 as a new USP22 interactor. Methods: We used western blotting and RT-PCR to measure key protein, acetylated STAT3, and mRNA levels in HEK293 and colorectal cancer cell lines transfected with expression plasmids or specific siRNAs. Co-immunoprecipitation was used to demonstrate protein-protein interaction and protein complex composition. Results: USP22 overexpression down-regulated STAT3 acetylation by deubiquitinating SIRT1. The three proteins were found to be present in a single protein complex. SiRNA-mediated depletion of endogenous USP22 resulted in SIRT1 destabilization and elevated STAT3 acetylation. Consistent with this finding, USP22 also down-regulated the expression of two known STAT3 target genes, MMP9 and TWIST. Conclusion: We show that USP22 is a new regulator of the SIRT1-STAT3 signaling pathway and report a new mechanistic explanation for cross talk between USP22 and the SIRT1-STAT pathways.
\end{abstract}

\section{Introduction}

Ubiquitin-specific peptidase 22 (USP22) is a subunit of the human Spt-Ada-Gen5 acetyltransferase (hSAGA) complex and a member of the deubiquitinating enzyme (DUB) family [1]. Within the hSAGA complex, USP22 deubiquitinates histones H2A and H2B to 


\section{Cellular Physiology and Biochemistry}

Cell Physiol Biochem 2014;33:1863-1875

\begin{tabular}{l|l}
\hline DOI: $10.1159 / 000362964$ & (C) 2014 S. Karger AG, Basel
\end{tabular}

www.karger.com/cpb

Ao et al.: USP22 Inhibits the STAT Signaling Pathway by Deubiquitinating SIRT1

regulate gene transcription and expression [2]. Far upstream element-binding protein 1 (FBP1) and telomeric repeat-binding factor 1 (TRF1) are deubiquitinated by USP22 [3, 4]. SIRT1 deubiquitination by USP 22 in HCT116 colon cancer cells leads to decreased p53 acetylation and the suppression of p53-mediated apoptosis [5]. Furthermore, USP22 located within the hSAGA transcriptional coactivating complex can be deacetylated by SIRT1. In addition, USP22-mediated recruitment of SIRT1 promotes the deacetylation of other components of hSAGA complex [6].

Sirtuins (Sirt proteins) are type III ( $\left.\mathrm{NAD}^{+}\right)$-dependent histone/protein deacetylases (HDACs) that control the packaging of DNA into chromatin and play critical roles in the regulation of gene expression [7]. SIRT1 deacetylates a variety of proteins including histones $\mathrm{H} 1, \mathrm{H} 3$, and H4, and may mediate heterochromatin formation [8]. Besides histones, several other proteins serve as SIRT1 substrates, including p53, c-Myc, FOXO, NF- $\mathrm{KB}$, and PGC-1 $\alpha$ [912]. In particular, SIRT1 mediates STAT3 lysine-685 deacetylation, which in turn negatively regulates STAT3 tyrosine-705 phosphorylation [13].

Members of the STAT protein family have been identified as latent cytoplasmic transcription factors that mediate cellular responses to cytokines, growth factors, and other polypeptide ligands $[14,15]$. Activation of STAT3 by phosphorylation is mediated by cytoplasmic kinases and growth factor receptor tyrosine kinases. Phosphorylated STAT3 is translocated from the cytoplasm to the nucleus, where it binds to specific DNA response elements and stimulates the expression of target genes [16]. STAT3 is involved in the oncogenesis of colorectal cancer and promotes the invasion of colon cancer cells. Interestingly, STAT3 phosphorylation and activity are tightly regulated by acetylation of lysine-685, located in the C-terminal transcriptional activation domain $[17,18]$. Steady state STAT3 acetylation depends on a tight balance between acetylation and deacetylation, primarily performed by p300 histone acetyltransferase and HDAC enzymes, respectively [17]. Furthermore, experiments using specific anti-acetylated lysine antibodies and a STAT3 mutant encoding a lysine- 685 to arginine substitution have revealed that STAT3 is acetylated at lysine- 685 by p 300 , and that acetylation at this site is critical for the formation of stable dimers required for transcriptional activity [18].

This study confirms that USP22 functions as a SIRT1 deubiquitinating enzyme. Our results show for the first time that USP22 negatively regulates STAT signaling pathway activation by SIRT1 and highlight a novel mechanism through which a ubiquitin peptidase mediates STAT3 deactivation.

\section{Materials and Methods}

\section{Reagents, Antibodies, and Plasmids}

Monoclonal anti-USP22 antibody was obtained from Epitomics (Burlingame, CA, USA). Anti-STAT3 and anti-acetyl-STAT3 (K685) antibodies were obtained from Cell Signaling Technology (Danvers, MA, USA). Polyclonal anti-SIRT1 and anti-TWIST1 antibodies were purchased from Proteintech Group Inc. (Chicago, IL, USA). Monoclonal anti-HA antibody was obtained from the Zhongshan Golden Bridge Co. Ltd (Beijing, China). Protein A/G Plus agarose was purchased from Santa Cruz Biotechnology (Santa Cruz, CA, USA). The FlagWT-USP22 expression plasmid and pcDNA $3.1(+)$ vector were gifts from Dr. Sharon Y. R. Dent (University of Texas M.D. Anderson Cancer Center, Houston, TX, USA). The SIRT1-WT expression plasmid was a gift from Dr. Ishikawa (Institute of Technology, Tokyo, Japan). The Flag-STAT3 and HA-Ub expression plasmids were gifts from Dr. Zhijie Chang (Tsinghua University, Beijing, China). The p300 expression plasmid was obtained from Dr. Ye Zhang (Chinese Academy of Medical Sciences and Peking Union Medical College, China).

\section{Cell Culture and Transfection}

Colon cancer cell lines HCT116 (adenocarcinoma) HT29 (adenocarcinoma), SW480 (adenocarcinoma) and human embryonic kidney cell line (HEK293) used in this study were obtained from the Cell Resource Center, Institute of Basic Medical Sciences, Chinese Academy of Medical Sciences, Peking Union Medical College (CRC- PUMC; Beijing, China); they were authenticated as mycoplasma free by short tandem repeat 
Ao et al.: USP22 Inhibits the STAT Signaling Pathway by Deubiquitinating SIRT1

Table 1. Primers used for quantitative reverse transcription PCR

\begin{tabular}{|c|c|}
\hline Primer name & Sequence \\
\hline \multirow{2}{*}{ USP22 } & Sense 5'-GACAACTGGAAGCAGAACC-3' \\
\hline & Antisense 5'-CGTACATCAGATCAATGGC-3' \\
\hline \multirow{2}{*}{ STAT3 } & Sense 5'-AACTCTTGGGACCTGGTGTG-3' \\
\hline & Antisense 5'-CGGACTGGATCTGGGTCTTA-3' \\
\hline \multirow{2}{*}{ MMP9 } & Sense 5'-TACCGAGAGAAAGCCTATT-3' \\
\hline & Antisense 5'-CACCTGGTTCAACTCACT-3' \\
\hline \multirow{2}{*}{ TWIST } & Sense 5'-GGAGTCCGCAGTCTTACGAG-3' \\
\hline & Antisense 5'-TCTGGAGGACCTGGTAGAGG-3' \\
\hline \multirow{2}{*}{ GAPDH } & Sense 5'-AGGTCGGTGTGAACGGATTTG-3' \\
\hline & Antisense 5'-TGTAGACCATGTAGTTGAGGTCA-3' \\
\hline
\end{tabular}

PCR. Cells were cultured as recommended conditions by the CRC-PUMC (http://cellresource.cn). HCT116, HT29 and HEK293 cells were cultured in Dulbecco's modified Eagle medium supplemented with 10\% fetal bovine serum (Gibco-BRL, Gaithersburg, MD). Another cell line, SW480, was maintained in Iscove's modified Dulbecco's medium containing $10 \%$ FBS. All cell lines were grown under $5 \% \mathrm{CO}_{2}$ at $37^{\circ} \mathrm{C}$ in a humidified environment. Transfections were performed according to the conventional standard transfection protocol (FuGENE HD, Roche, Mannheim, Germany).

\section{Immunoprecipitation and Immunoblotting}

Cells transfected with various combinations of plasmids DNA were lysed with RIPA Buffer (50 mM Tris- $\mathrm{HCl}, \mathrm{pH}$ 8, $130 \mathrm{mM} \mathrm{NaCl}, 1 \% \mathrm{NP}-40,0.5 \%$ sodium deoxycholate, and 0.2\% SDS), supplemented with protease inhibitor (Amresco, Solon, OH, USA) and phenylmethylsulfonyl fluoride (Beyotime Institution of Biotechnology, Haiman China). Immunoprecipitations were performed at $4^{\circ} \mathrm{C}$ using the appropriate antibodies and protein A/G-agarose (Santa Cruz, CA, USA). Protein samples were separated by $10 \%$ SDS-PAGE (Beyotime Institution of Biotechnology, Haimen, China) and transferred onto nitrocellulose membranes (Pall Gelman Laboratory, Stevenage, UK). For immunodetection, blots were incubated with primary antibodies overnight at $4^{\circ} \mathrm{C}$, and then washed and incubated with a 1:5000 dilution of horseradish peroxidase (HRP)-conjugated anti-mouse IgG [HtL (heavy chain t light chain); Zhongshan Golden Bridge Biotechnology Co. Ltd, Beijing, China] or a 1:3000 dilution of HRP-conjugated anti-rabbit IgG (HtL; Zhongshan Golden Bridge Biotechnology Co. Ltd) for 1 h. ECL Super Signal West Pico Chemiluminescent Substrate (Pierce, Appleton, WI, USA) was used to visualize the protein bands. To determine protein levels, the optical density of each protein band was analyzed using a Quantity One 1-D Analysis System (Bio-Rad Laboratories Inc., Hercules, CA, USA).

\section{RNA Silencing}

The siRNA method was used to knock down USP22 expression in HCT116, HT29 and SW480 cells. USP22 siRNA was obtained from RiboBio Co. (Guangzhou, China): the sequence was 5'-GGAGAAAGAUCACCUCGAAdTdT-3'. STAT3 siRNA was obtained from Invitrogen: the sequence was 5'-GGAAAGAAGGAAUAUCUCA dTdT-3'. Ncontrol_05815 (NCsi; RiboBio Co, Guangzhou, China) was used as a negative control for all siRNA experiments. Transfection was performed using Lipofectamine 2000 (Invitrogen, Carlsbad, CA, USA) according to the manufacturer's instructions.

\section{RNA Isolation and Real-Time RT-PCR}

Total RNA was extracted using the TRIzol reagent (Life Technologies Corporation, Carlsbad, CA, USA). Reverse transcription (RT) was performed using a PrimeScript RT Master Mix kit (TAKARA Biotechnology Co. Ltd., Dalian, China). The cDNA products were subjected to real-time PCR using a SYBR Premix Ex TaqTM II kit (TAKARA Biotechnology Co. Ltd.). Real-time PCR was performed using a StepOnePlus Real-Time PCR System (Applied Biosystems, Carlsbad, CA, USA) using the following program: $95^{\circ} \mathrm{C}$ for $10 \mathrm{~min}$, followed by 40 cycles of $95^{\circ} \mathrm{C}$ for $15 \mathrm{~s}$ and $60^{\circ} \mathrm{C}$ for $1 \mathrm{~min}$. Each sample was analyzed in triplicate. GAPDH mRNA was quantified and used as an endogenous control. The primer sequences used in this study are shown in Table 1. 


\section{Cellular Physiology and Biochemistry}

Cell Physiol Biochem 2014;33:1863-1875

\begin{tabular}{l|l}
\hline DOI: $10.1159 / 000362964$ & (C) 2014 S. Karger AG, Basel
\end{tabular}

Publisned oninne: June 23, 2014

www.karger.com/cpb

Ao et al.: USP22 Inhibits the STAT Signaling Pathway by Deubiquitinating SIRT1

\section{Zymography}

MMP9 proteolytic activity in cell lysates was determined by gelatin zymography. Twenty-four hours after siRNA transfection, transfection medium was removed and cells were incubated in serum-free medium for a further $48 \mathrm{~h}$. Cell lysates were then subjected to electrophoresis on 10\% SDS-polyacrylamide gels containing $0.1 \%$ gelatin. Gels were incubated twice for $45 \mathrm{~min}$ at room temperature in $2.5 \%$ Triton X-100 (Qxbio, Beijing, China), and then incubated in activation buffer $\left(50 \mathrm{mmol} / \mathrm{L}\right.$ Tris-Cl pH8.0, $10 \mathrm{mmol} / \mathrm{L} \mathrm{CaCl}_{2}$, $50 \mathrm{mmol} / \mathrm{L} \mathrm{NaCl}$ ) for $42 \mathrm{~h}$ at $37^{\circ} \mathrm{C}$. Gels were then stained with $0.05 \%$ Coomassie Brilliant Blue R-250 for $3 \mathrm{~h}$ and destained sequentially in wash buffer I (10\% acetic acid, 30\% methanol), II (10\% acetic acid, $20 \%$ methanol), and III ( $5 \%$ acetic acid, $10 \%$ methanol) for $0.5,1$, and $2 \mathrm{~h}$, respectively. Regions of gelatinolytic activity were detected as transparent bands on a blue background.

\section{Immunocytochemistry}

Cells were fixed in 4\% paraformaldehyde in phosphate-buffered saline (PBS) for 30 min and preincubated in blocking buffer (PBS containing 10\% normal rabbit serum) for $30 \mathrm{~min}$. Cells were then incubated at $4^{\circ} \mathrm{C}$ overnight in primary antibody (anti-MMP9, 1:300; Bioworld, China). After washing with PBS, cells were incubated with fluorescein isothiocyanate (FITC)-labeled rabbit anti-goat IgG secondary antibody (1:200; Zhongshan Golden Bridge Biotechnology Co. Ltd) at $37^{\circ} \mathrm{C}$ for $1 \mathrm{~h} .4^{\prime}, 6$-diamidino-2phenylindole was used for counterstaining. Photomicrographs were captured with an Olympus IX51 (Miyazaki, Japan).

\section{Statistical Analysis}

Data are expressed as the mean \pm SD. Statistical analyses to compare pairs of experimental groups were performed using the Student's t-test or the two-sample t-test with SPSS version 13.0 (Statistical Package for the Social Sciences) statistics software. The threshold for statistical significance was defined as $P<0.05$.

\section{Results}

USP22 Interacts with STAT3 and Inhibits STAT3 Acetylation Mediated by SIRT1 Deubiquitination

USP22 deubiquitinates SIRT1, and SIRT1 is known to inhibit STAT3 acetylation. We therefore hypothesized that USP22 may inhibit STAT3 acetylation through deubiquitinating SIRT1. To test this hypothesis, we first investigated whether SIRT1 is ubiquitinated in cells using methods described previously [5]. In fact, a high molecular weight protein ladder was detected in anti-SIRT1 immunoprecipitates using anti-HA antibodies (Fig. 1A), indicating that SIRT1 is polyubiquitinated. A small amount of ubiquitinated SIRT1 is present in HEK293 cells without SIRT1-WT cotransfection, suggesting that a low level of endogenous SIRT1 ubiquitination exists in HEK293 cells. Cotransfection of WT USP22 and SIRT1-WT significantly inhibited ubiquitin conjugation to SIRT1 relative to transfection with SIRT1-WT alone, thus confirming that USP22 deubiquitinates SIRT1 in HEK293 cells.

We next investigated a possible interaction between USP22 and STAT3 in HEK293 cells. USP22 and STAT3 proteins were overexpressed, cell lysates were immunoprecipitated using an anti-USP22 antibody, and interacting proteins were identified by western blotting. A STAT3/USP22 interaction was shown by anti-USP22 antibody pull down and immunoblotting using an anti-STAT3 antibody (Fig. 1B). A small amount of STAT3 was pulled down by USP22 in the absence of WT USP2 2 cotransfection, suggesting that STAT3 interacts with endogenous USP22 in HEK293 cells. However, WT USP22 and Flag-STAT3 cotransfection significantly enhanced the USP22/STAT3 interaction, confirming that USP22 interacts with STAT3 in HEK293 cells. To rule out a nonspecific interaction, control IgG antibody was used instead of anti-USP22 antibody. USP2 2 did not bind to control IgG, indicating that USP22 specifically binds to STAT3.

As USP22 is a ubiquitin-specific protease, we next investigated whether USP22 deubiquitinates STAT3 by examining STAT3 ubiquitination levels using the methods already described. A high molecular weight protein ladder was detected in anti-STAT3 


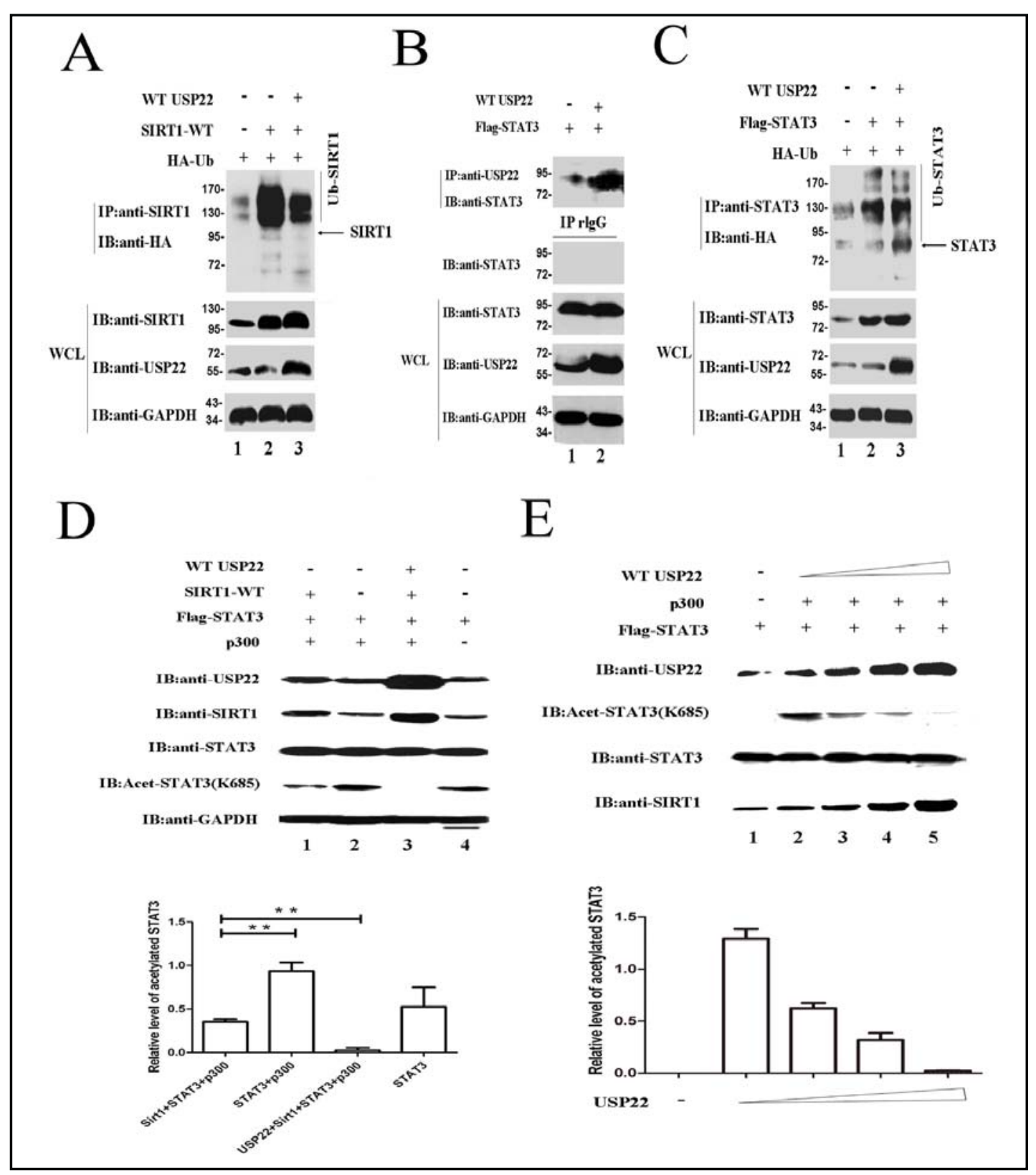

Fig. 1. USP22 inhibits STAT3 acetylation by deubiquitinating SIRT1. (A) SIRT1 ubiquitination was detected by SIRT1 immunoprecipitation followed by western blotting with an anti-HA antibody (top panel). Levels of SIRT1 (second panel), USP22 (third panel), and GAPDH (fourth panel) expression in HEK293 whole-cell lysates (WCL) are shown following HA-ubiquitin transfection (lane 1), SIRT1-WT and HA-ubiquitin cotransfection (lane 2), and SIRT1-WT, HA-ubiquitin, and WT USP22 cotransfection (lane 3). pcDNA 3.1 was the negative control for WT USP22. (B) The USP22/STAT3 interaction was demonstrated by USP22 immunoprecipitation and western blotting with an anti-STAT3 antibody (top panel). Immunoprecipitation with an anti-IgG antibody and western blotting with anti-STAT3 antibody (second panel) served as a control. Levels of STAT3 (third panel) and USP22 (fourth panel) expression in HEK293 whole-cell lysates (WCL) are shown following Flag-STAT3 transfection (lane 1) and Flag-STAT3 and WT USP22 cotransfection (lane 2). pcDNA 3.1 was the control plasmid for WT USP22. (C) STAT3 ubiquitination was detected by STAT3 immunoprecipitation followed by western blotting with an anti-HA antibody (top panel). Levels of STAT3 (second panel), USP22 (third panel), and GAPDH (fourth panel) expression in HEK293 whole-cell lysates (WCL) are shown following HA-ubiquitin transfection (lane 1), Flag-STAT3 and HA-Ubiquitin cotransfection (lane 2), and Flag-STAT3, HA-Ubiquitin, and WT USP22 cotransfection (lane 3). pcDNA 3.1 was the control plasmid 
for WT USP22. (D) Levels of USP22, SIRT1, STAT3, acetylated STAT3, and GAPDH expression were assessed by western blotting after cotransfection of different combinations of plasmids into HEK293 cells: FlagSTAT3 transfection (lane 4); SIRT1-WT, Flag-STAT3, and p300 cotransfection (lane 1); Flag-STAT3 and p300 cotransfection (lane 2); and WT USP22, SIRT1-WT, Flag-STAT3, and p300 cotransfection (lane 3). STAT3 acetylation was detected with an anti-acetyl-STAT3 (K685) antibody and quantified by densitometry. ${ }^{* *} P<$ 0.01. (E) Levels of USP22, SIRT1, STAT3, and acetylated STAT3 expression were assessed by western blotting after increasing amounts of WT USP22, Flag-STAT3, and p300 plasmids (lanes 1-5) were cotransfected into HEK293 cells. pcDNA 3.1 was the control plasmid for WT USP22. STAT3 acetylation was quantified by densitometry.

Fig. 2. USP22 inhibition of STAT3 acetylation is mediated by SIRT1. (A) The USP22/STAT3 interaction was detected by of USP22 immunoprecipitation followed by western blotting with an antiSTAT3 antibody (top panel) after cotransfection of different combinations of plasmids into HT29 (left panel) and SW480 cells (right panel). Levels of USP22 (second panel), SIRT1 (third panel), and STAT3 (fourth panel) expression in HT29 and SW480 wholecell lysates (WCL) are shown following FlagSTAT3 transfection (lane 2), Flag-STAT3 WT USP22 cotransfection (lane 3), and WT USP22, SIRT1WT, and Flag-STAT3 cotransfection (lane 4). Controls without WT USP22 and FlagSTAT3 transfection are shown (lane 1). pcDNA 3.1 was the

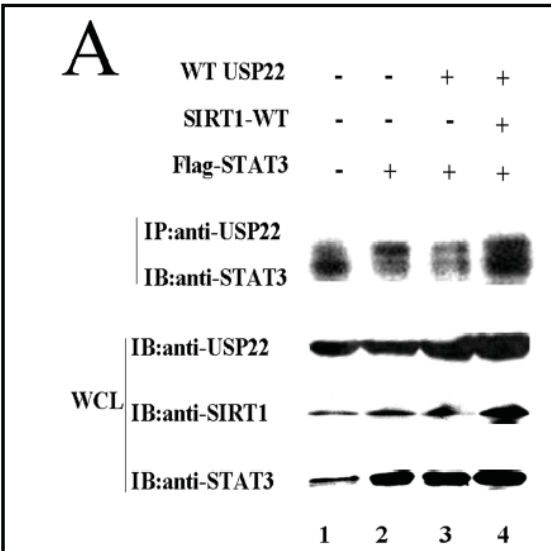

B

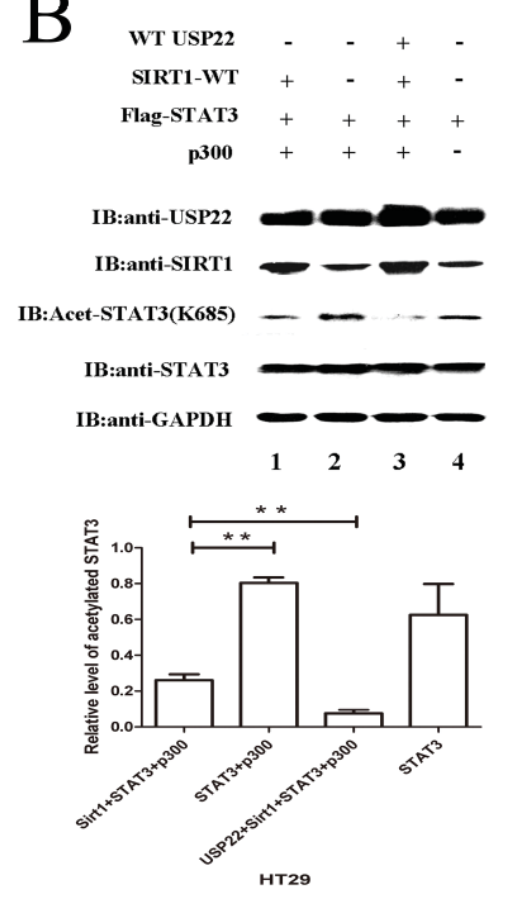

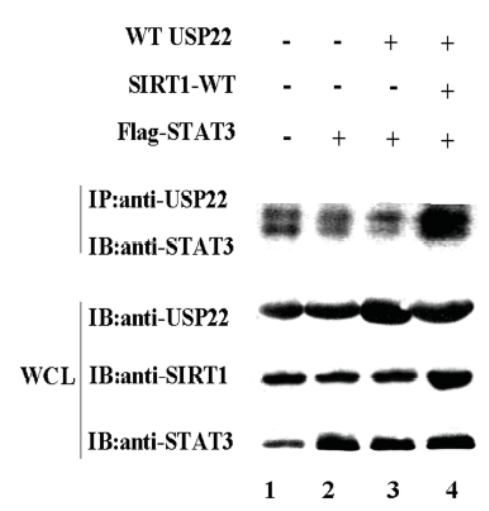
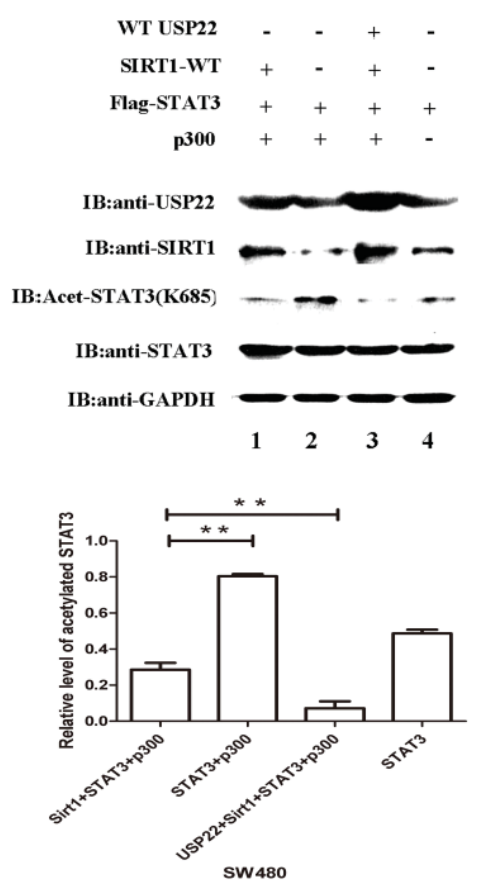

control plasmid for WT USP22. (B) Levels of USP22, SIRT1, STAT3, acetylated STAT3, and GAPDH expression were analyzed in HT29 and SW480 cells by western blotting after cotransfection of different combinations of plasmids into HT29 (left panel) and SW480 cells (right panel): Flag-STAT3 transfection (lane 4); SIRT1-WT, Flag-STAT3, and p300 cotransfection (lane 1); Flag-STAT3 and p300 cotransfection (lane 2), and WT USP22, SIRT1-WT, Flag-STAT3, and p300 cotransfection (lane 3). STAT3 acetylation was quantified by densitometry. ${ }^{* *} P<0.01$. 


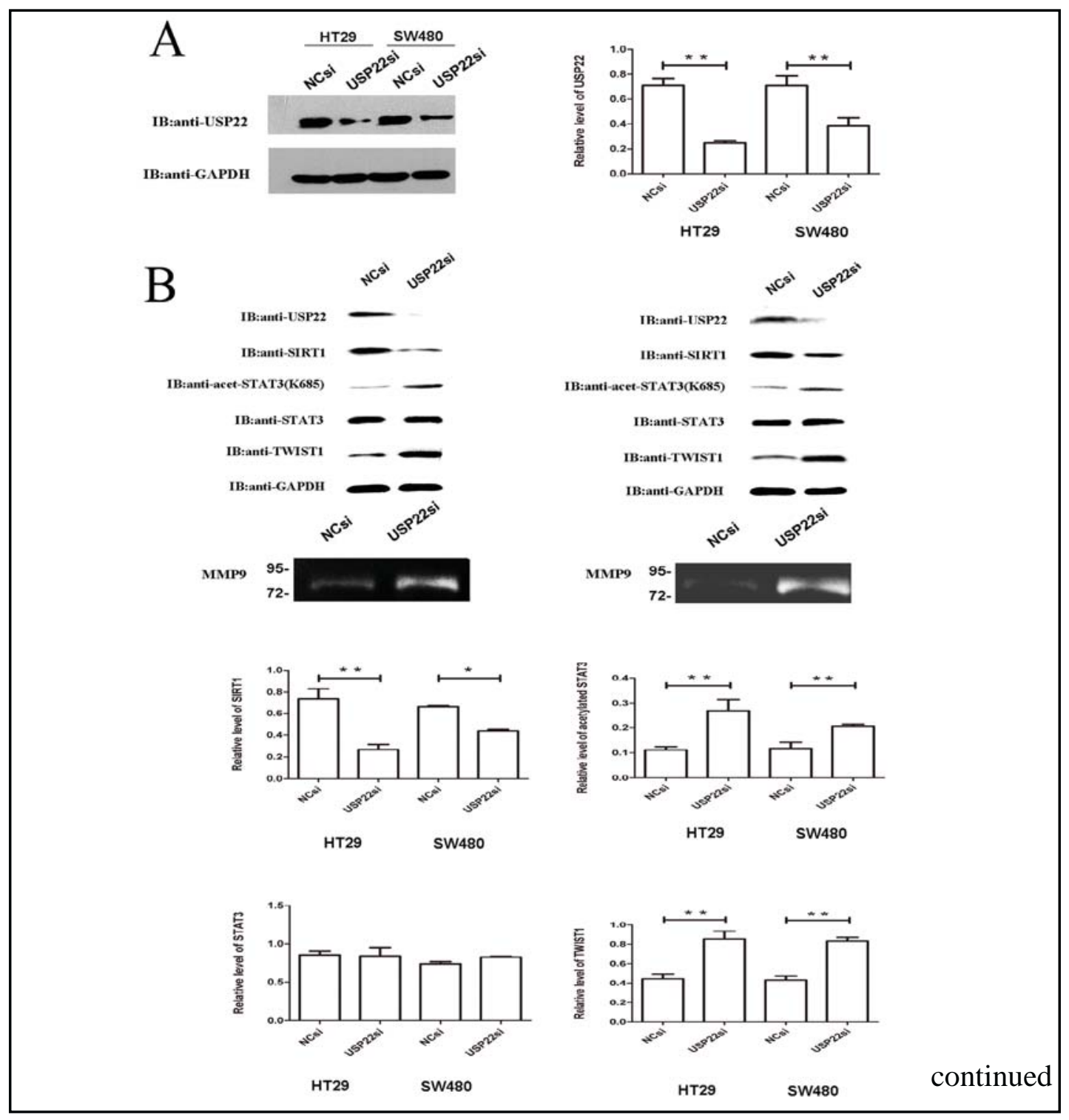

Fig. 3. USP2 2 depletion alters the expression of STAT3 target genes by altering the SIRT1-STAT3 pathway in HT29 and SW480 cells. (A) The proteins were isolated from HT29 and SW480 cells transfected with control (NCsi) or USP22 (USP22si) siRNA. The expression of USP22 was analyzed by western blotting. USP22 was quantified by densitometry. ${ }^{* *} P<0.01$. (B) HT29 (left panel) and SW480 cells (right panel) were transfected with control (NCsi) or USP22 (USP22si) siRNA, and the expression of USP22, SIRT1, STAT3, and TWIST1 proteins and acetylated STAT3 were determined by western blotting. GAPDH was used as the loading control. SIRT1, STAT3 acetylation, STAT3 and TWIST1 were quantified by densitometry. ${ }^{*} P<0.05, * * P<0.01$. A zymography assay was used to measure the proteolytic activity of MMP9 in HT29 (left panel) and SW480 cells (right panel) transfected with control (NCsi) or USP22 (USP22si) siRNA. (C) Total RNA was isolated from HT29 (top panel) and SW480 cells (bottom panel) transfected with control (NCsi) or USP22 (USP22si) siRNA. Levels of USP22, STAT3, and STAT3 target gene (MMP9, and TWIST) mRNA in HT29, SW480 cells were determined by real-time reverse transcription PCR. ${ }^{*} P<0.01$. (D) Indirect immunofluorescence of MMP9 in HT29 (left panel) and SW480 cells (right panel) transfected with NCsi or USP22 siRNA. MMP9-positive cells were identified using a fluorescein isothiocyanate-labeled antibody. Cell nuclei were identified by 4',6diamidino-2-phenylindole staining.

immunoprecipitates following immunoblotting with anti-HA antibodies (Fig. 1C), indicating that STAT3 is polyubiquitinated. A small amount of ubiquitinated STAT3 is present in HEK293 


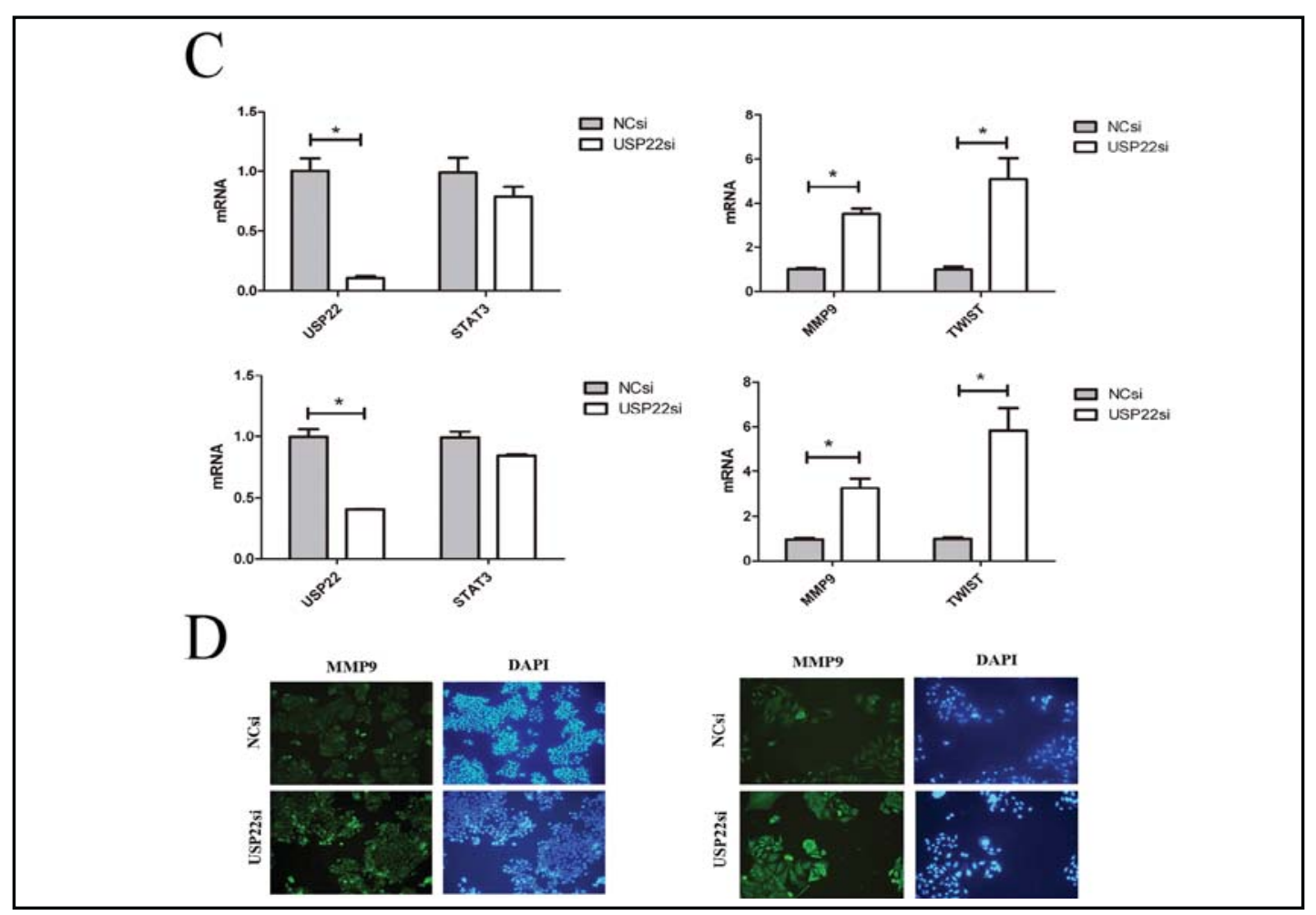

cells in the absence of Flag-STAT3 cotransfection, suggesting that some ubiquitination of endogenous STAT3 occurs in HEK293 cells. USP22 cotransfection did not affect ubiquitin conjugation to STAT3, thus confirming that USP22 does not deubiquitinate STAT3 in HEK293 cells.

Finally, to test whether USP22 induces STAT3 deacetylation by SIRT1, we investigated STAT3 acetylation in HEK293 cells cotransfected with different combinations of USP22, SIRT1, STAT3, and p300 expression plasmids. We found that SIRT1 overexpression inhibits STAT3 acetylation, and this was enhanced by USP22 and SIRT1 coexpression (Fig. 1D). Following transfection of increasing amounts of WT USP22, endogenous SIRT1 protein levels increased, STAT3 acetylation decreased, but total STAT3 protein remained unchanged. In fact, USP22 deacetylated STAT3 without affecting of STAT3 expression, and this effect was associated with increased SIRT1 levels. Thus, there was a positive correlation between USP22 and SIRT1 expression, and an inverse relationship between both USP22 and SIRT1 expression and STAT3 acetylation (Fig. 1E).

Moreover, to investigate their interaction, USP22 and STAT3 were overexpressed in HT29 and SW480 in colon cancer cells. USP22 was immunoprecipitated from cell lysates and interacting proteins were identified by western blotting. The STAT3/USP22 interaction was shown by USP22 immunoprecipitation followed by immunoblotting using an anti-STAT3 antibody. A USP22/STAT3 interaction was detected in untransfected HT29 and SW480 cells, suggesting that USP22 interacts with endogenous STAT3. However, neither Flag-STAT3 transfection nor WT USP22 and Flag-STAT3 cotransfection affected the endogenous USP22/ STAT3 interaction, suggesting that the endogenous USP22/STAT3 interaction is strong in HT29 and SW480 cells (Fig. 2A). The interaction between USP22 and STAT3 was increased in SIRT1-overexpressing cells, but not in USP22- or STAT3-overexpressing cells. Therefore, the interaction of USP22 with STAT3 is mediated by SIRT1. SIRT1 is reported to reduce STAT3 acetylation $[12,13]$. To verify that USP22 affects STAT3 acetylation by deubiquitinating SIRT1, we cotransfected different combinations of USP22, SIRT1, STAT3, and p300 expression plasmids into HT29 and SW480 cells. SIRT1 inhibition of STAT3 acetylation was enhanced by USP22 coexpression (Fig. 2B). Therefore, USP22 suppression of STAT3 acetylation, a posttranslational modification essential for STAT3 function, is mediated by SIRT1. 


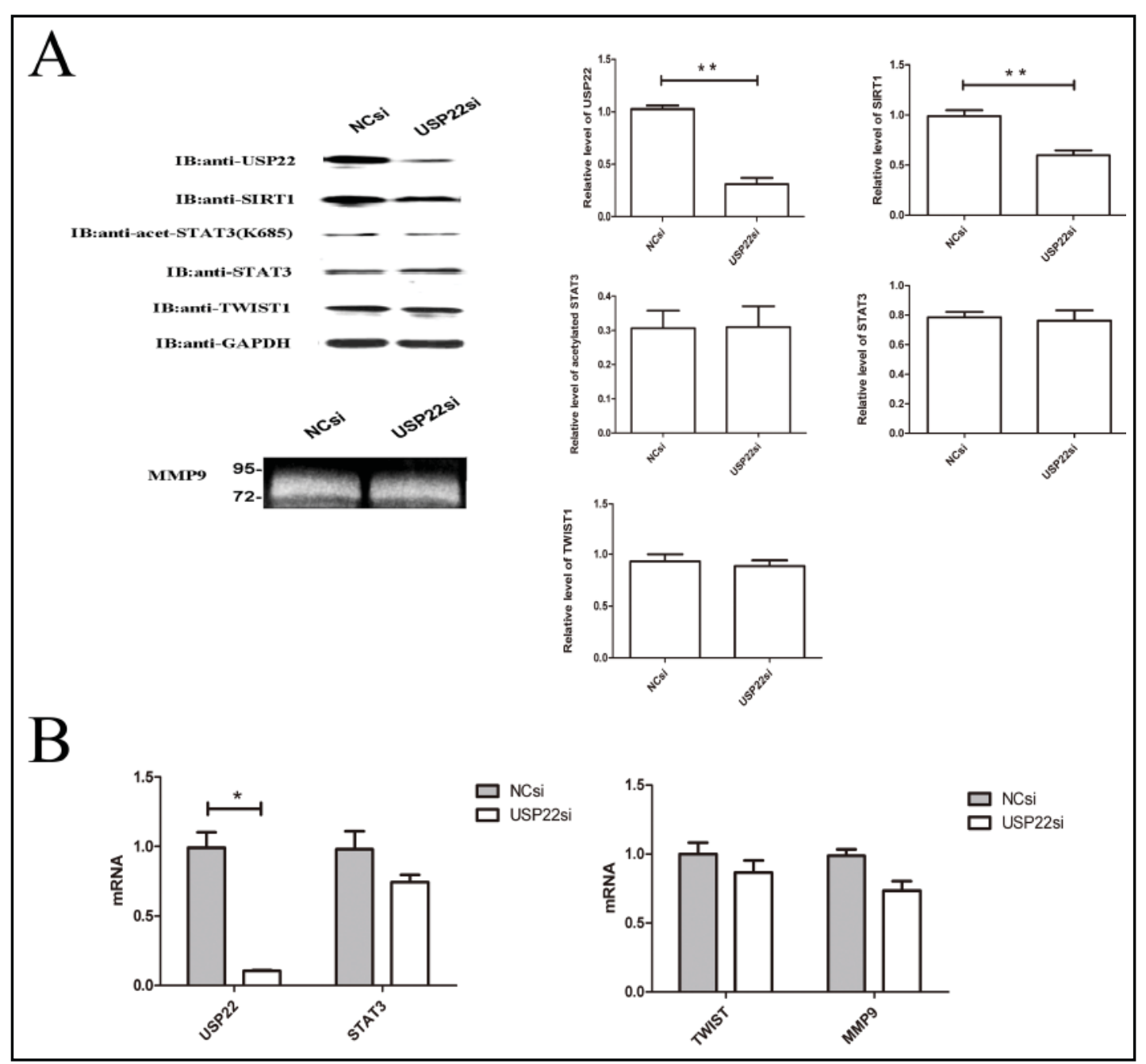

Fig. 4. USP22 depletion does not affect STAT3 acetylation and expression of STAT3 target genes in HCT116 cells. (A) HCT116 cells were transfected with control (NCsi) or USP22 (USP22si) siRNA, and the expression of USP22, SIRT1, STAT3, and TWIST1 proteins and acetylated STAT3 were determined by western blotting. GAPDH was used as the loading control. USP22, SIRT1, STAT3 acetylation, STAT3 and TWIST1 were quantified by densitometry. ${ }^{* *} P<0.01$. A zymography assay was used to measure the proteolytic activity of MMP9 in HCT116 cells transfected with control (NCsi) or USP22 (USP22si) siRNA. (B) Total RNA was isolated from HCT116 cells (bottom panel) transfected with control (NCsi) or USP22 (USP22si) siRNA. Levels of USP22, STAT3, and STAT3 target gene (MMP9, and TWIST) mRNA in HCT116 cells were determined by real-time reverse transcription PCR. ${ }^{*} P<0.01$.

USP22 Downregulates Expression of STAT3 Target Genes by SIRT1-STAT3 Pathway

We next investigated USP22 function in colon cancer cells using siRNA-mediated gene silencing. We first showed that the USP22 protein levels were decreased $48 \mathrm{~h}$ after transfection with USP22 siRNA relative to treatment with control siRNA in HT29, SW480, HCT116 cells. We show that USP22 knockdown in HT29 and SW480 cells reduces SIRT1 protein levels and elevates endogenous STAT3 acetylation, without affecting STAT3 protein levels (Fig. 3A,B), thus confirming that USP22 posttranscriptionally regulates STAT3. We next examined the effects of USP22 knockdown on the expression of STAT3 mRNA and of transcripts of the STAT3 target genes MMP9 and TWIST $[19,20]$. Both MMP9 mRNA levels and proteolytic activity were significantly increased in HT29 and SW480 cells; similarly, TWIST mRNA and protein levels were significantly increased in HT29 and SW480 cells (Fig. 3B,C). In addition, USP22 silencing led to consistently higher levels of MMP9 protein in HT29 and SW480 cells, 


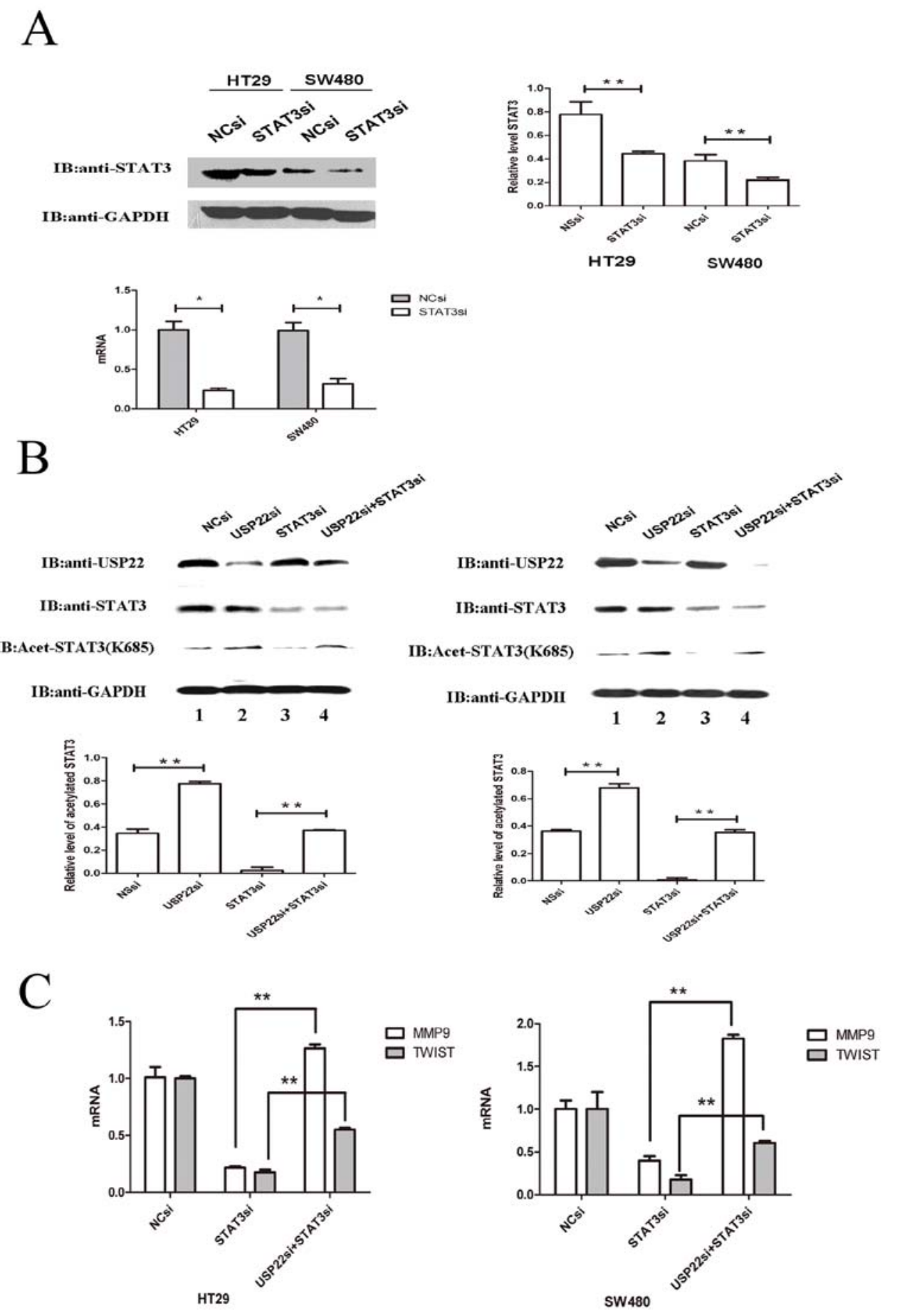

Fig. 5. USP22 inhibits STAT3 function by deacetylating STAT3 in HT29 and SW480 cells. (A) Western blot (top panel) and real-time reverse transcription PCR (bottom panel) analyses show STAT3 protein and mRNA expression in HT29 and SW480 cells $48 \mathrm{~h}$ after transfection with control (NCsi) or STAT3 (STAT3si) siRNA. STAT3 protein expression was quantified by densitometry. ${ }^{* *} P<0.01$. (B) HT29 (left panel) and SW480 cells (right panel) were transfected with NCsi (lane 1), USP22si (lane 2), STAT3 si (lane 3) or a combination of USP22si/STAT3si (lane 4). Expression levels of USP22 and STAT3 protein and acetylated STAT3 were determined by western blotting. GAPDH was used as a loading control. STAT3 acetylation was quantified by densitometry. ${ }^{* * P}<0.01$. (C) Expression of MMP9 and TWIST mRNA following STAT3 or combined USP22 and STAT3 depletion in HT29 (left panel) and SW480 cells (right panel). ${ }^{* *} P<0.01$. 


\section{Cellular Physiology and Biochemistry}

Cell Physiol Biochem 2014;33:1863-1875

DOI: 10.1159/000362964

Publisned oninne: June 23,2014

Ao et al: USP22 Inhibits the STAT Sig

as demonstrated by indirect immunofluorescence (Fig. 3D). However, USP22 knockdown in HCT116 cells reduces SIRT1 protein levels, without affecting endogenous STAT3 acetylation and STAT3 mRNA and protein levels (Fig. 4A,B). Similarly, MMP9 and TWIST expression were not affected by USP22 knockdown in HCT116 cells (Fig. 4A,B).

\section{USP22 Represses STAT3 Transcriptional Activity by Supressing STAT3 Acetylation}

USP22 overexpression suppresses STAT3 acetylation by deubiquitinating SIRT1. USP22 depletion upregulated STAT3 target genes MMP9 and TWIST through the SIRT1-STAT3 pathway. Furthermore, siRNA transfection of HT29 and SW480 cells showed that STAT3 mRNA and protein levels were decreased $48 \mathrm{~h}$ after STAT3 siRNA transfection relative to levels in control siRNA-treated cells (Fig. 5A). To test the effect of simultaneous USP22 and STAT3 knockdown, we measured STAT3 acetylation and MMP9 and TWIST mRNA expression after combined USP22 and STAT3 siRNA treatment compared with STAT3 siRNA alone in HT29 and SW480 cells. USP22 co-depletion partly rescued reduced STAT3 acetylation, as well as MMP9 and TWIST mRNA levels, induced by STAT3 depletion alone (Fig. 5B,C). We consider that hyperacetylation of the remaining STAT3 protein in the absence of USP22 can lead to transcriptional activation of these STAT3 target genes.

In conclusion, our studies demonstrate that USP22-mediated SIRT1 deubiquitination inhibits STAT3 acetylation and its transcriptional activation.

\section{Discussion}

USP22 stabilizes SIRT1 by removing the polyubiquitin chains that target SIRT1 for proteasome-mediated degradation. USP22-mediated stabilization of SIRT1 leads to reduced levels of p53 acetylation and the suppression of p53 transcription activation [5]. SIRT1 also deacetylates STAT3 at lysine- 685 and, in turn, negatively regulates STAT3 phosphorylation at tyrosine-705 $[13,18]$. Therefore, SIRT1-mediated STAT3 deacetylation may inhibit the sequence-specific DNA binding ability and transactivation activity of STAT3. However, whether USP22-SIRT1 pathways affect STAT3 activation is unclear.

Lysine-685 acetylation of STAT3 is inhibited by both interleukin-22 (IL-22) and SIRT1, and SIRT1 opposes IL-22-induced STAT3 activation by deacetylating STAT3 and reducing STAT3 tyrosine-705 phosphorylation [13]. By controlling STAT3 dimerization, SIRT1 also influences IL-22-induced upregulation of molecules involved in the proliferation and inflammation of cultured keratinocytes. Therefore, it is possible that USP22 regulates STAT3 activity by deubiquitinating SIRT1. However, prior to this study it was unknown whether (1) USP22 interacts with STAT3 and (2) this interaction is mediated by SIRT1.

Cleavage of ubiquitin moieties from target substrates by the DUB enzyme USP22 is a key element in many physiological and pathological processes. There is increasing evidence that USP22 can efficiently remove ubiquitin from the core histones H2A and H2B in vitro and thus regulate gene transcription $[21,22]$. We show for the first time that the ubiquitin peptidase USP22 binds to STAT3 in mammalian cells in vitro. USP22 is also reported to bind to and deubiquitinate SIRT1, a transcription factor responsible for STAT3 dimerization. Our data demonstrate that USP22 suppresses STAT3 acetylation by deubiquitinating SIRT1, without affecting STAT3 ubiquitination. This suggests that USP22 inhibits STAT3 dimerization to regulate STAT3 activity. We also examined the effect of USP2 2 depletion on STAT3-dependent target gene transcription in HT29 and SW480 cells, and found upregulation of two STAT3 target genes following USP22 knockdown.

USP22 has been showed to stabilize SIRT1 in HCT116 cells. In this study, we found SIRT1 to be downregulated by USP22 knockdown in HCT116, HT29, and SW480 cells. However, there were differences in the levels of STAT3 acetylation in these three cell lines after USP22 knockdown: STAT3 acetylation was increased in HT29 and SW480 cells, but not in HCT116 cells (Fig. 4A). Different genetic mutations may account for this difference. For example, HT29 cells contain mutated B-RAF, SW480 cells have mutated K-RAS, and HCT116 


\section{Cellular Physiology and Biochemistry}

Cell Physiol Biochem 2014;33:1863-1875

\begin{tabular}{l|l}
\hline DOI: $10.1159 / 000362964$ & (c) 2014 S. Karger AG, Basel
\end{tabular}

www.karger.com/cpb

Ao et al.: USP22 Inhibits the STAT Signaling Pathway by Deubiquitinating SIRT1

cells have mutated K-RAS and mutated PI3K [23]. Ohbayashi et al. found STAT3 lysine-685 acetylation to be suppressed by the PI3K inhibitor, LY294002, or by dominant negative Akt in 293T and Hep3B cells [24]. Further studies proved that endogenous STAT3 is acetylated at lysine- 685 by leukemia inhibitory factor (LIF) or IL-6 through activation of the PI3K-Akt pathway. Therefore, PI3K mutation in HCT116 cells may suppress STAT3 acetylation through the PI3K-Akt pathway. In addition, we found no change in STAT3 acetylation levels after USP22 knockdown in HCT116 cells. Therefore, the effects of USP22 depletion on MMP9 and TWIST expression are most likely a result of hyperacetylation of the remaining STAT3 in HCT116 cells (Fig. 4A,B).

USP22 can remove ubiquitin moieties from histones H2A and H2B in vitro. This ability led to an initial hypothesis that the function of USP22 is linked to the regulation of gene transcription. USP22 was shown to affect the expression of c-Myc target genes, and its depletion leads to the accumulation of cells in the G1 phase of the cell cycle. Atanassov et al. observed that the abundant ubiquitination of FBP1 upon USP22 ablation ensures the activation of target loci, such as MMP1, in normal human fibroblasts and HeLa cells [3]. That observation led us to show that MMP9 inhibition by USP22 occurs via STAT3 deacetylation in HT29 and SW480 cells.

Direct deubiquitination of transcriptional factors plays a key role in the activation of several signaling pathways. In its role as a ubiquitin peptidase, USP22 binds to the STAT3 transcription factor and suppresses STAT3 acetylation by deubiquitinating SIRT1. This study therefore provides evidence of a functional interaction between USP22 and STAT3. The detailed molecular mechanisms by which USP22 deactivates the STAT3 pathway should be investigated further, as should its biological roles in the antiviral response, immune and inflammatory responses, and cancer.

\section{Acknowledgments}

The authors appreciate the gifts of plasmids from Dr. Sharon Y.R. Dent (University of Texas M.D. Anderson Cancer Center, Houston, TX, USA), Dr. Ishikawa (Institute of Technology, Tokyo, Japan), Dr. Zhijie Chang (Tsinghua University, Beijing China), and Dr. Ye Zhang (Chinese Academy of Medical Sciences and Peking Union Medical College, China).

\section{References}

1 Zhang XY, Pfeiffer HK, Thorne AW, McMahon SB: USP22, An hSAGA subunit and potential cancer stem cell marker,reverses the polycomb-catalyzed ubiquitylation of histone H2A. Cell Cycle 2008;7:1522-1524.

-2 Lang G, Bonnet J, Umlauf D, Karmodiya K, Koffler J, Stierle M, Devys D, Tora L: The tightly controlled deubiquitination activity of the human SAGA complex differentially modifiesdistinct gene regulatory elements. Mol Cell Biol 2011;31:3734-3744.

-3 Atanassov BS, Dent SY: USP22 regulates cell proliferation by deubiquitinating the transcriptional regulator FBP1. Embo Reports 2011;12:924-930.

4 Atanassov BS1, Evrard YA, Multani AS, Zhang Z, Tora L, Devys D, Chang S, Dent SY: Gcn5 and SAGA regulate shelterin protein turnover and telomere maintenance. Mol Cell 2009;35:352-364.

-5 Lin Z, Yang H, Kong Q Li J, Lee SM, Gao B, Dong H, Wei J, Song J, Zhang DD, Fang D: USP22 antagonizes p53 transcriptional activation by deubiquitinating SIRT1 to suppress cell apoptosis and is required for mouse embryonic development. Mol Cell 2012;46:484-494.

6 Armour SM, Bennett EJ, Braun CR, Zhang XY, McMahon SB, Gygi SP, Harper JW, Sinclair DA: A highconfidence interaction map identifies SIRT1 as a mediator of acetylation of USP22 and the SAGA coactivator complex. Mol Cell Biol 2013;33:1487-1502. 


\section{Cellular Physiology and Biochemistry}

Cell Physiol Biochem 2014;33:1863-1875

-7 Smith JS, Brachmann CB, Celic I, Kenna MA, Muhammad S, Starai VJ, Avalos JL, Escalante-Semerena JC, Grubmeyer C, Wolberger C, Boeke JD: A phylogenetically conserved NAD+-dependent protein deacetylase activity in the Sir2 protein family. Proc Natl Acad Sci 2000;97:6658-6663.

8 Luo K, Vega-Palas MA, Grunstein M: Rap1-Sir4 binding independent of other Sir, yKu, or histone interactions initiates the assembly of telomeric heterochromatin in yeast. Genes Dev 2002;16:1528-1539.

-9 Langley E, Pearson M, Faretta M, Bauer UM, Frye RA, Minucci S, Pelicci PG, Kouzarides T: Human SIR2 deacetylates p53 and antagonizes PML/p53-induced cellular senescence. EMBO J 2002;21:2383-2396.

10 Yuan J, Minter-Dykhouse K,Lou Z: A c-Myc-SIRT1 feedback loop regulates cell growth and transformation. J Cell Biol 2009;185:203-211.

11 Motta MC, Divecha N, Lemieux M, Kamel C, Chen D, Gu W, Bultsma Y, McBurney M, Guarente L: Mammalian SIRT1 represses forkhead transcription factors. Cell 2004;116:551-563.

12 Zhang J, Lee SM, Shannon S, Gao B, Chen W, Chen A, Divekar R, McBurney MW, Braley-Mullen H, Zaghouani H, Fang D: The type III histone deacetylase SIRT1 is essential for maintenance of T cell tolerance in mice. J Clin Invest 2009;119:3048-3058.

13 Sestito R, Madonna S, Scarponi C, Cianfarani F, Failla CM, Cavani A, Girolomoni G, Albanesi C: STAT3dependent effects of IL-22 in human keratinocytes are counterregulated by sirtuin 1 through a directinhibition of STAT3 acetylation. FASEB J 2011;25:916-927.

14 Darnell JE Jr, Kerr IM, Stark GR: Jak-STAT pathways and transcriptional activation in response to IFNs and other extracellular signaling proteins. Science 1994;264:1415-1421.

15 Zhong Z, Wen Z, Darnell JE Jr: Stat3: a STAT family member activated by tyrosine phosphorylation in response to epidermal growth factor and interleukin-6. Science 1994;264:95-98.

-16 Dauer DJ, Ferraro B, Song L, Yu B, Mora L, Buettner R, Enkemann S, Jove R, Haura EB: Stat3 regulates genes common to both wound healing and cancer. Oncogene 2005;24:3397-3408.

17 Yuan ZL, Guan YJ, Chatterjee D, Chin YE: Stat3 dimerization regulated by reversible acetylation of a single lysine residue. Science 2005;307:269-273.

18 Nie Y, Erion DM, Yuan Z, Dietrich M, Shulman GI, Horvath TL, Gao Q: STAT3 inhibition of gluconeogenesis is downregulated by SIRT. Nat Cell Biol 2009;11:492-450.

19 Alvarez JV, Frank DA: Genome-wide analysis of STAT3 target genes: elucidating the mechanism of STATmediated oncogenesis. Cancer Biol Ther 2004;3:1045-1050.

20 Cheng GZ, Zhang WZ, Sun M, Wang Q Coppola D, Mansour M, Xu LM, Costanzo C, Cheng JQ Wang LH: Twist is transcriptionally induced by activation of STAT3 and mediates STAT3 oncogenic function. J Biol Chem 2008;283:14665-14673.

-21 Zhang XY, Varthi M, Sykes SM, Phillips C, Warzecha C, Zhu W, Wyce A, Thorne AW, Berger SL, McMahon SB:. The putative cancer stem cell marker USP22 is a subunit of the human SAGA complex required for activated transcription and cell-cycle progression. Mol Cell 2008;29:102-111.

-22 Chipumuro E, Henriksen MA.The ubiquitin hydrolase USP22 contributes to 3'-end processing of JAK-STATinducible genes. FASEB J 2012;26:842-854.

23 Li ZW, Liu YQ: Progress of targeted therapy related to K-ras mutation. Zhong Hua Bing Li Xue Za Zhi 2012;41:59-61.

24 Ohbayashi N, Ikeda O, Taira N, Yamamoto Y, Muromoto R, Sekine Y, Sugiyama K, Honjoh T, Matsuda T: LIF- and IL-6-induced acetylation of STAT3 at Lys-685 through PI3K/Akt activation. Biol Pharm Bull 2007;30:1860-1864. 